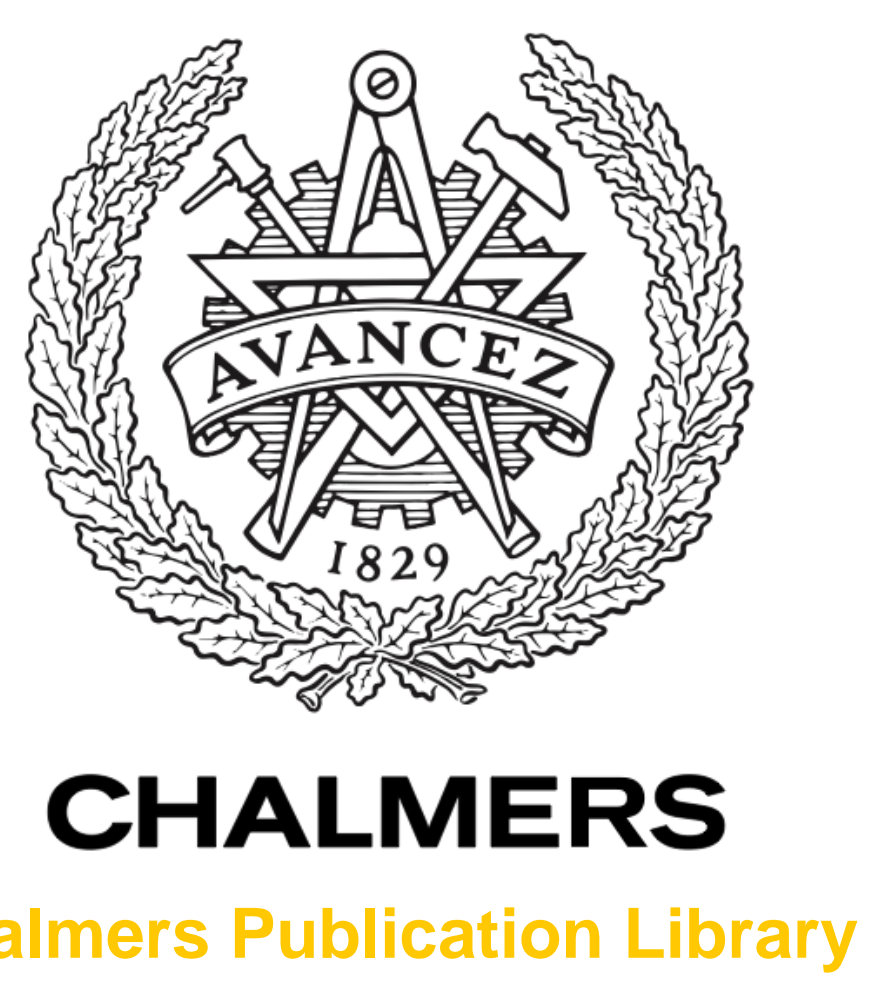

Chalmers Publication Library

\title{
Reducing the spectral width of high speed oxide confined VCSELs using an integrated mode filter
}

This document has been downloaded from Chalmers Publication Library (CPL). It is the author's version of a work that was accepted for publication in:

Vertical-Cavity Surface-Emitting Lasers Xvi (ISSN: 0277-786X)

Citation for the published paper:

Haglund, E. ; Haglund, A. ; Gustavsson, J. et al. (2012) "Reducing the spectral width of high speed oxide confined VCSELs using an integrated mode filter". Vertical-Cavity Surface-

Emitting Lasers Xvi, vol. 8276 pp. Article Number: 82760L.

http://dx.doi.org/10.1117/12.908424

Downloaded from: http://publications.lib.chalmers.se/publication/156115

Notice: Changes introduced as a result of publishing processes such as copy-editing and formatting may not be reflected in this document. For a definitive version of this work, please refer to the published source. Please note that access to the published version might require a subscription.

Chalmers Publication Library (CPL) offers the possibility of retrieving research publications produced at Chalmers University of Technology. It covers all types of publications: articles, dissertations, licentiate theses, masters theses, conference papers, reports etc. Since 2006 it is the official tool for Chalmers official publication statistics. To ensure that Chalmers research results are disseminated as widely as possible, an Open Access Policy has been adopted.

The CPL service is administrated and maintained by Chalmers Library. 


\title{
Reducing the spectral width of high speed oxide confined VCSELs using an integrated mode filter
}

\author{
Erik Haglund*a, Åsa Haglund ${ }^{\mathrm{a}}$, Johan S. Gustavsson ${ }^{\mathrm{a}}$, Benjamin Kögel ${ }^{\mathrm{a}}$, Petter Westbergh ${ }^{\mathrm{a}}$ \\ and Anders Larsson ${ }^{\mathrm{a}}$ \\ ${ }^{a}$ Department of Microtechnology and Nanoscience, Photonics Laboratory, \\ Chalmers University of Technology, Göteborg SE-41296, Sweden
}

\begin{abstract}
We have reduced the spectral width of high speed oxide confined $850 \mathrm{~nm}$ VCSELs using a shallow surface relief for suppression of higher order transverse modes. The surface relief acts as a mode filter by introducing a spatially varying and therefore mode selective loss. The VCSEL employs multiple oxide layers for reduced capacitance which leads to a strong index guiding and a large spectral width in the absence of a mode filter. With an appropriate choice of surface relief parameters, the RMS spectral width for a $5 \mu \mathrm{m}$ oxide aperture VCSEL is reduced from 0.6 to $0.3 \mathrm{~nm}$. The small signal modulation bandwidth is $19 \mathrm{GHz}$. Due to reduced effects of chromatic and modal fiber dispersion, the maximum error-free (bit-error-rate $<10^{-12}$ ) transmission distance at $25 \mathrm{~Gb} / \mathrm{s}$ over OM3 + fiber is increased from 100 to $500 \mathrm{~m}$.
\end{abstract}

Keywords: VCSEL, transverse modes, spectral width, mode filter, surface relief, high speed modulation

\section{INTRODUCTION}

Vertical-cavity surface-emitting lasers (VCSELs) are today well established light sources for a number of applications in communication and sensing thanks to advantages such as low fabrication cost, circular output beam characteristics, fast direct modulation at low currents and low power consumption [1]. GaAs-based high speed multimode VCSELs for short-reach optical communication at $850 \mathrm{~nm}$ have seen an impressive increase in bandwidth over the last couple of years, reaching small signal modulation bandwidths of $23 \mathrm{GHz}$ and enabling transmission at $40 \mathrm{~Gb} / \mathrm{s}$ back-to-back [2]. However, for transmission distances of a few hundred meters over multimode OM3 + fiber, the increased impact of modal and chromatic fiber dispersion due to the multiple transverse VCSEL modes will reduce the transmission quality at bit rates above $10 \mathrm{~Gb} / \mathrm{s}[\underline{3}]$.

Here we present our recent work on extending the error-free transmission distance with oxide confined AlGaAs $850 \mathrm{~nm}$ VCSELs at bit rates as high as $25 \mathrm{~Gb} / \mathrm{s}$. A mode filter was etched into the top distributed Bragg reflector (DBR), introducing a spatially varying and therefore mode selective loss, which enhances the fundamental mode and suppresses higher order modes. The VCSELs employing the mode filter show a 50\% reduction in root-mean-square (RMS) spectral width, from 0.6 to $0.3 \mathrm{~nm}$, and increased maximum error-free transmission distance from 100 to $500 \mathrm{~m} \mathrm{OM} 3$ + fiber.

\section{VCSEL DESIGN AND TRANSVERSE MODE PROPERTIES}

\subsection{High speed VCSEL design}

The high speed VCSEL structure is shown in Figure 1. It is grown by metal-organic chemical vapor deposition on semiinsulating GaAs. The DBRs have graded interfaces and modulation doping to reduce resistance and most of the bottom n-DBR contains binary AlAs as the low refractive index material to improve thermal conductivity. Five strained InGaAs/AlGaAs quantum wells are employed for high differential gain and the separate confinement heterostructure is designed for fast carrier capture and low gain compression [4]. Two selectively oxidized $\mathrm{Al}_{0.98} \mathrm{Ga}_{0.02} \mathrm{As}_{\text {s layers located at }}$ an antinode of the optical field provide current confinement and index guiding, while four shallow $\mathrm{Al}_{0.96} \mathrm{Ga}_{0.02} \mathrm{As}$ oxide layers help to reduce the mesa capacitance, mitigating the parasitic impairments [5]. A thick layer of benzocyclobutene $(\mathrm{BCB})$ is used to planarize the structure and reduce bondpad capacitance. The top p-DBR has an anti-phase top surface

*erik.haglund@chalmers.se; phone: +46(0)31 772 5481; www.chalmers.se/mc2

Vertical-Cavity Surface-Emitting Lasers XVI, edited by Chun Lei, Kent D. Choquette, Proc. of SPIE Vol. 8276, 82760L · @ 2012 SPIE · CCC code: 0277-786X/12/\$18 · doi: 10.1117/12.908424 
reflection to provide a short photon lifetime for enhanced modulation bandwidth [6]. The anti-phase layer also facilitates the integration of a surface relief mode filter [7].

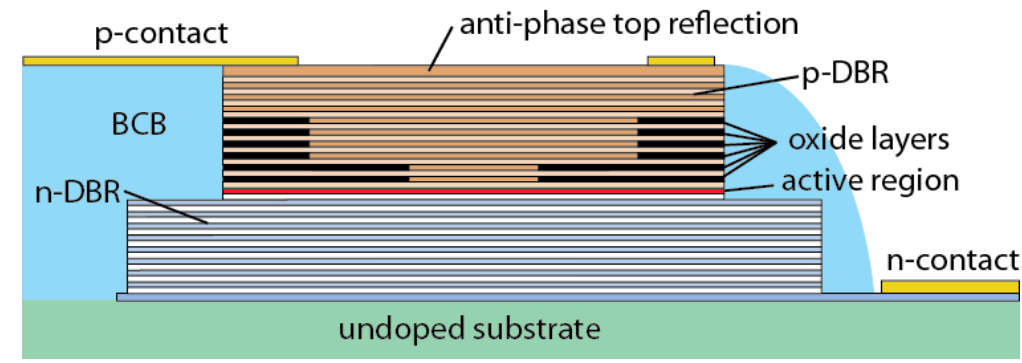

Figure 1. Cross sectional view of the high speed VCSEL design.

\subsection{Transverse mode properties}

Large transverse dimensions in combination with a strong index guiding from the multiple oxide layers make these VCSELs transversely multimode. Due to the circular symmetry of the VCSELs, these modes closely resemble linearly polarized $\left(\mathrm{LP}_{m n}\right)$ optical fiber modes [1]. The different transverse intensity distributions of the modes and the resonant requirement cause a blueshift of the higher order transverse modes [8]. Figure 2 shows a typical spectrum and spectrally resolved near-field images of the lowest order transverse modes. The different intensity distributions and the corresponding different wavelengths will deteriorate the quality of a high frequency signal transmitted through an optical fiber by modal and chromatic dispersion, respectively. It is therefore of great importance to reduce the spectral width to reduce the effects of chromatic dispersion and reduce the number of transverse modes to reduce the effects of modal dispersion. To quantify the spectral width of a VCSEL, the root mean square (RMS) spectral width is calculated according to

$$
\Delta \lambda_{\text {RMS }}=\sqrt{\sum_{i=1}^{n} \frac{P_{i}}{P_{\text {tot }}}\left(\lambda_{i}-\lambda_{\text {mean }}\right)^{2}}, \quad \lambda_{\text {mean }}=\sum_{i=1}^{n} \frac{P_{i}}{P_{\text {tot }}} \lambda_{i}
$$

where $P_{i}$ is the power in point $i$ of the measured spectrum, $P_{\text {tot }}$ is the total power, $\lambda_{i}$ is the wavelength of point $i$ and $\lambda_{\text {mean }}$ is the average wavelength. Our high speed VCSELs have RMS spectral widths of up to $1.2 \mathrm{~nm}$, which is far beyond that specified in the IEEE-803.2ae standard (10 Gbit/s over up to $300 \mathrm{~m}$ OM3 fiber), which requires an RMS width $<0.45 \mathrm{~nm}$. It is clear that the spectral width of the VCSEL must be reduced to reach longer transmission distances and fulfill requirements in upcoming standards.
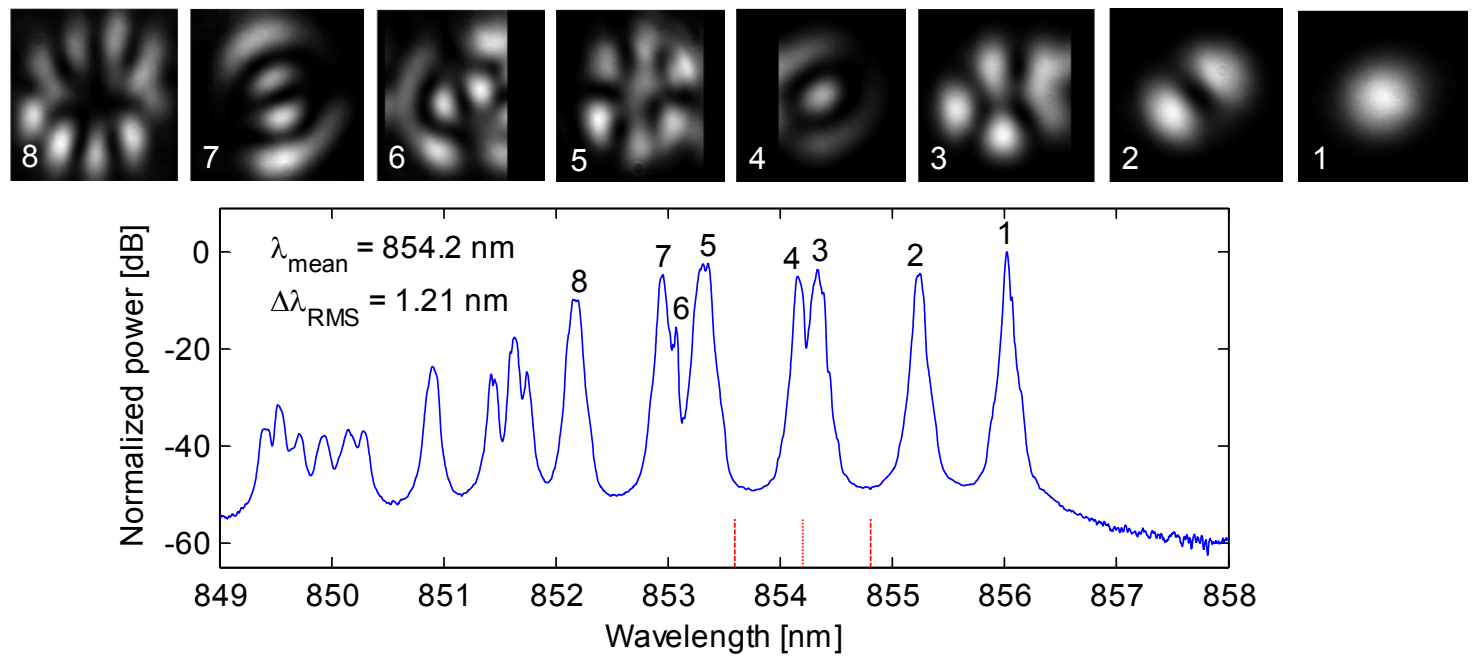

Figure 2. Emission spectrum recorded for an anti-phase top reflection VCSEL with an oxide aperture of $7 \mu \mathrm{m}$ driven at $7 \mathrm{~mA}$. The mean wavelength and RMS spectral width are marked on the x-axis. Above: Spectrally resolved near-field images showing the intensity distribution of the first 8 transverse modes as marked in the spectrum. 


\section{SURFACE RELIEF MODE FILTER}

The most straightforward way to reduce the spectral width is to reduce the diameter of the oxide aperture, which will eventually result in a single mode VCSEL [9]. A small oxide aperture, however, leads to higher differential resistance and thereby increased self-heating. Other techniques to reduce spectral width include metal apertures [10], extended cavities [11], curved mirrors (980 nm, bottom emitting) [12] and complex structures such as photonic crystals [13]. Most of these methods have the disadvantages of adding considerable complexity to device design and fabrication and may not be suitable for high speed direct modulation.

\subsection{Surface relief method}

One way to reduce the spectral width, without directly influencing the electrical and thermal properties, is to prevent higher order modes from reaching lasing threshold by introducing mode selective loss. The top mirror loss can be greatly varied by etching into the uppermost layer of the top DBR, as seen in Figure $3 \mathrm{~b}$ ). Since the transverse modes of the VCSEL have different radial intensity distributions, see Figure $3 \mathrm{c}$ ), a spatially varying top DBR loss can be used to prevent all or some of the higher order modes from reaching lasing threshold. This is achieved by etching a circular surface relief (SR) in the top DBR as illustrated by A and B in Figure 3 a) - c). The use of an anti-phase reflection design relaxes the required precision in etch depth [7]. Etching to an in-phase reflection in the center of the waveguide lowers the loss for the lowest order transverse modes, thereby suppressing higher order modes.

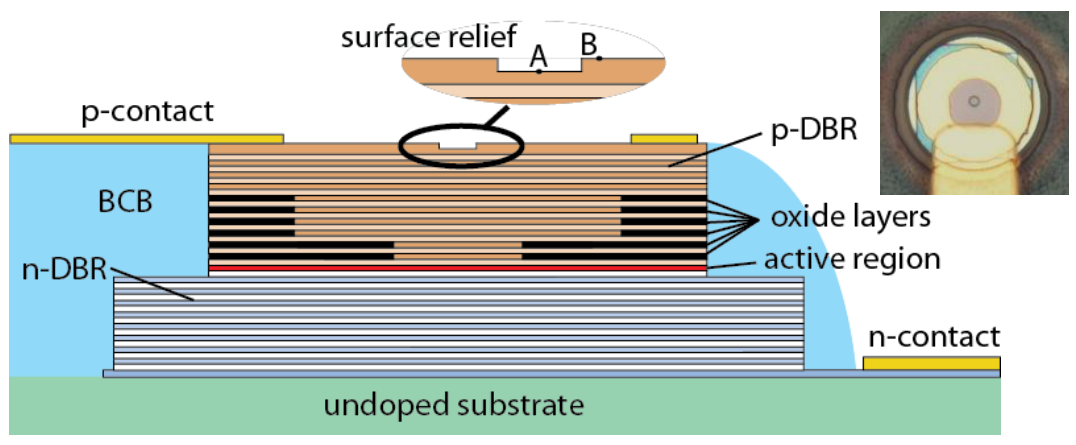

a)

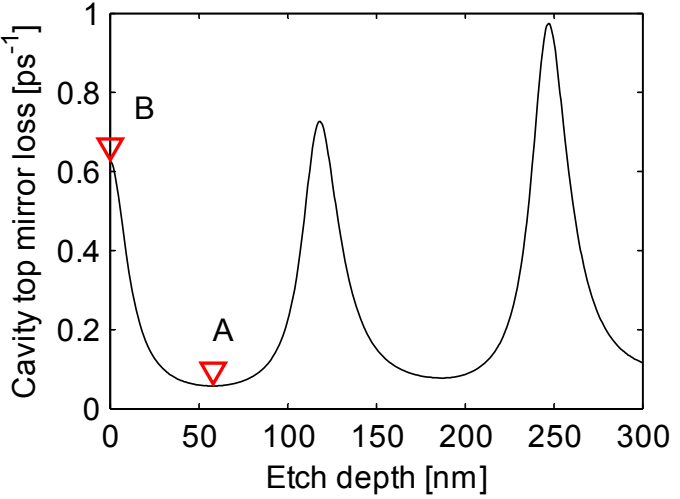

b)

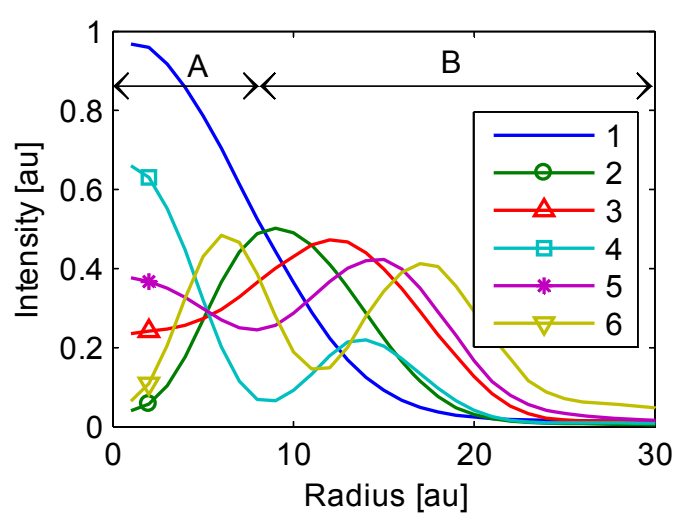

c)

Figure 3. a) Cross sectional view of the high speed VCSEL design with a shallow surface relief mode filter. Inset: microscope image of an SR-VCSEL. b) Calculated top mirror loss as a function of etch depth for the anti-phase design. c) Average radial intensity variations from the near-field images in Figure 2. The A and B mark the low and high top DBR loss regions forming the SR mode filter. 


\subsection{Fabrication}

The surface relief etch constitutes only a small modification to the standard VCSEL fabrication process. Electron beam lithography was used to define the surface relief in order to facilitate a precise alignment between the surface relief and the oxide aperture. The diameter of the surface relief is $1.5 \mu \mathrm{m}$. Argon ion milling was used to remove the $59 \mathrm{~nm}$ thick anti-phase layer, yielding a very precise and accurate etch depth. A microscope image of the finished SR-VCSEL can be seen in Figure 3 a).

\section{EXPERIMENTAL RESULTS}

Three VCSELs with different top surfaces were compared to evaluate the impact of the SR; one with the as-grown antiphase top DBR, one where a $1.5 \mu \mathrm{m}$ diameter surface relief was etched in the anti-phase layer and one where the topmost anti-phase layer was removed over the entire surface, giving an overall in-phase reflection from the top DBR. The oxide aperture diameter is $5 \mu \mathrm{m}$ for all VCSELs.

\subsection{Static measurements}

Figure 4 shows the output power and voltage versus bias current for the three VCSELs, measured at room temperature by positioning a large area detector close to the VCSEL. The modifications of the top mirror loss by removing the antiphase layer on parts of the surface (SR-VCSEL) or the entire surface (in-phase VCSEL) are seen to have a significant influence on the static properties. When the anti-phase layer is etched away, the top mirror reflectivity is increased. Since the anti-phase layer is removed only partly for the SR-VCSEL, it has an average top mirror reflectivity between that of the anti-phase and in-phase VCSELs. As the mirror reflectivity increases, both threshold current (inset in Figure 4) and slope efficiency decreases, as expected. Also, the thermal roll-over current is reduced since the longer photon lifetime results in a higher photon density in the cavity and therefore higher internal optical loss [14]. The slightly differing differential resistances of 165,179 and $173 \Omega$ for the anti-phase, SR and in-phase VCSEL, respectively, can be accounted for by small differences in oxide aperture diameter for the different components (estimated to $<0.2 \mu \mathrm{m}$ ).
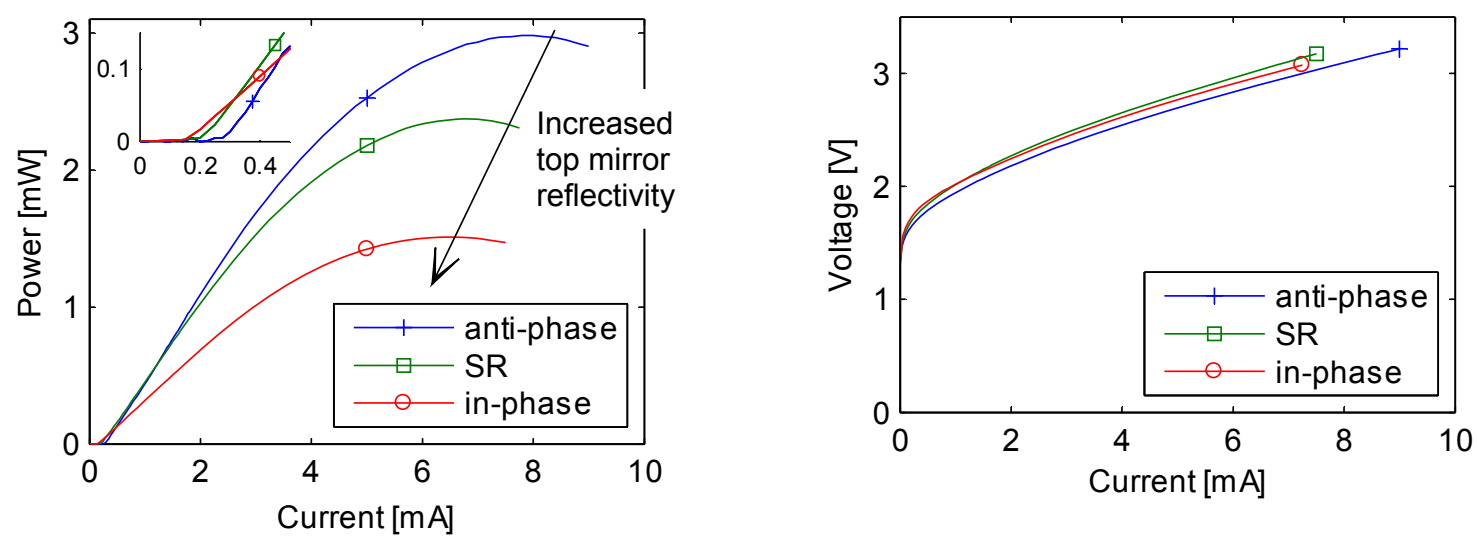

Figure 4. Left: Output power versus bias current. The inset shows a magnification of the threshold region. Right: Voltage versus bias current.

Figure 5 shows the optical spectra and RMS spectral width for the three VCSELs. The spectra were measured with an Ando AQ6317 optical spectrum analyzer by butt-coupling a short OM3+ optical fiber to the VCSEL. A significant reduction in RMS spectral width by more than $50 \%$ was seen for the SR-VCSEL compared to the anti-phase and inphase VCSELs. The mode spacing between the fundamental mode $\left(\mathrm{LP}_{01}\right)$ and the first higher order mode $\left(\mathrm{LP}_{11}\right)$ for the three lasers are $1.4 \pm 0.1 \mathrm{~nm}$, indicating that the VCSELs have similar guiding and therefore very similar oxide aperture sizes. This enables a fair evaluation of the influence of the SR mode filter.

A mode spacing of $1.4 \mathrm{~nm}$ is rather large, indicating a large amount of index guiding, which likely originates from the use of multiple oxide layers. In previous work, a rule of thumb has been that the surface relief diameter should equal half the oxide aperture diameter for optimal mode filtering [7]. Due to the stronger index guiding observed in these VCSELs, the modes are better confined to the center of the waveguide and a smaller surface relief with a diameter of only $30 \%$ of 
the oxide aperture gives optimal performance. The fact that the modes are spatially closer to each other in the center of the waveguide also makes it more difficult to filter out only the fundamental mode. The strong guiding in combination with non-uniform current injection explains why single mode lasing is not observed. Many VCSELs with slightly larger oxide apertures of $7 \mu \mathrm{m}$ even have a stronger $\mathrm{LP}_{11}$ than $\mathrm{LP}_{01}$ mode due to the strong index guiding and non-uniform current injection. Internal heating (thermal lensing) is not believed to have any significant influence on the guiding since the mode spacing increases only very slightly $(<0.1 \mathrm{~nm})$ from a bias current of $1 \mathrm{~mA}$ to roll-over.

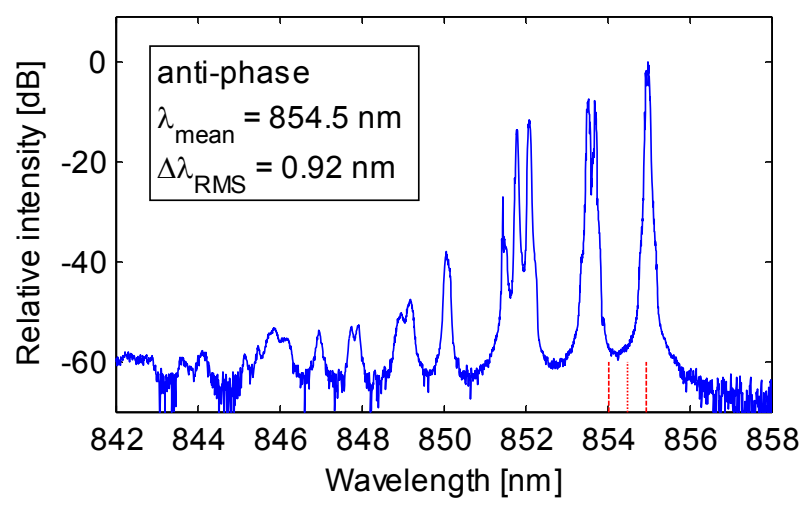

a)

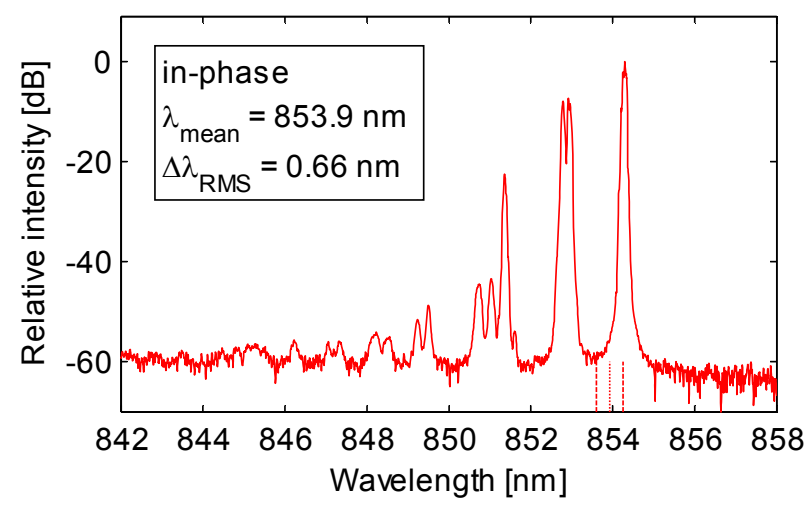

c)

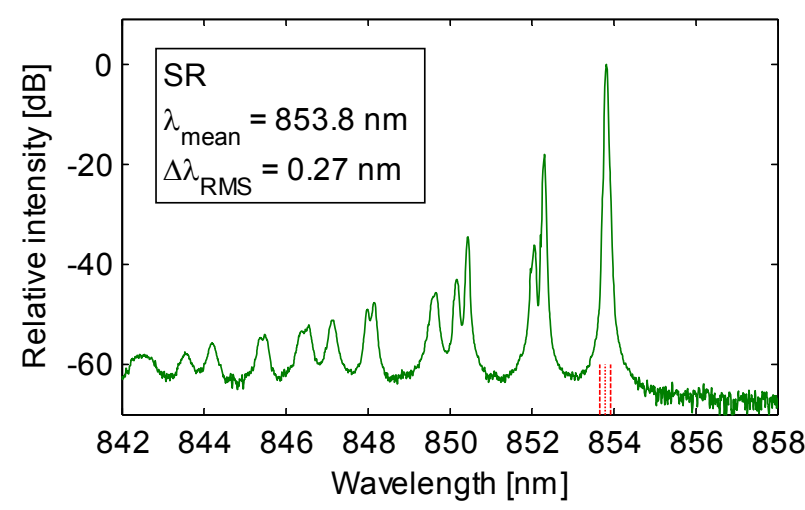

b)

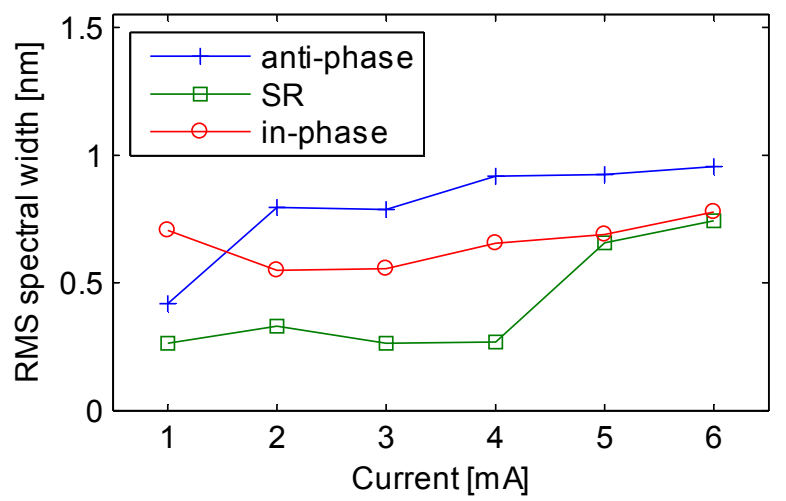

d)

Figure 5. Room temperature optical spectra at $4 \mathrm{~mA}$. The mean wavelength and RMS width are indicated on the $\mathrm{x}$-axes. a) Anti-phase VCSEL, b) SR-VCSEL and c) in-phase VCSEL. d) The RMS spectral width as a function of bias current.

\subsection{Dynamic measurements}

The dynamic properties of the VCSELs were quantified by measuring the small signal modulation response (S21) using a $65 \mathrm{GHz}$ Anritsu 37397C network analyzer. The signal was fed to the VCSEL through a bias-T and a high-frequency RF probe. An anti-reflection coated lens system and an angled facet fiber was used capture the output light, while minimizing optical feedback from the measurement setup. A $25 \mathrm{GHz}$ NewFocus 1481-S-50 photodetector was used to convert the optical signal back to the electrical domain before feeding it back to the network analyzer. The measured data was corrected for the photodetector response and probe insertion loss.

Since the top DBR reflectivity has a large impact on the photon lifetime, it also influences the damping of the modulation response. A lower reflectivity gives a shorter photon lifetime and reduced damping. Hence, reducing the top DBR reflectivity can extend the bandwidth of the VCSEL [6]. This behavior is seen in Figure 6; the anti-phase VCSEL has a $3 \mathrm{~dB}$ bandwidth of $22 \mathrm{GHz}$, while the SR and in-phase VCSELs reach 19 and $15 \mathrm{GHz}$, respectively. This can be viewed as a trade-off between the mode selective and high speed properties of the SR; good filtering requires a lower modal loss for the fundamental mode, giving a longer photon lifetime, while a relatively short photon lifetime is desired for good high speed properties. 


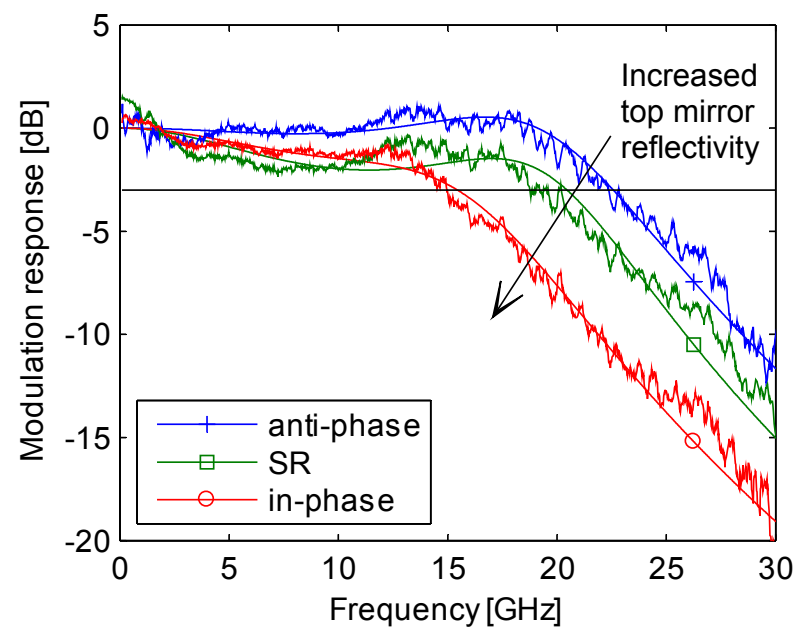

Figure 6. Measured small signal modulation response of the three VCSELs, at bias currents for maximum bandwidth.

\subsection{Transmission experiments}

Transmission experiments were carried out to verify the improvement of link performance by using SR-VCSELs with reduced spectral width. An SHF 12103A bit pattern generator was used to generate a $900 \mathrm{mV}_{\mathrm{pp}}$ non-return-to-zero pseudorandom binary sequence of $2^{7}-1$ bits. The same probe, fiber coupling setup and photodetector was used as in the small signal modulation measurements. The modulated optical signal was either transmitted through OM3+ high-speed multimode optical fiber (4700 MHz-km effective modal bandwidth) or connected back-to-back (BTB). A JDSU OLA-54 optical attenuator was inserted before the photodetector for the bit-error-rate (BER) measurements. The photodetector was directly followed by an SHF 804TL amplifier (22 dB gain, $55 \mathrm{GHz}$ bandwidth) to boost the signal. Eye diagrams were recorded using a $70 \mathrm{GHz}$ Agilent $86100 \mathrm{C}$ oscilloscope with a precision timebase. BERs were measured using an SHF 11100B error analyzer. Error-free operation is here defined as BER $<10^{-12}$. All transmission measurements were carried out at room temperature using data rates of $25 \mathrm{~Gb} / \mathrm{s}$.

Figure 7 shows the results of the $25 \mathrm{~Gb} / \mathrm{s}$ transmission experiments. The SR and in-phase VCSELs were biased at 3.5 $\mathrm{mA}$. The anti-phase VCSEL was biased at $5.5 \mathrm{~mA}$, since the shorter photon lifetime requires a larger current to establish the photon density needed for good high speed performance [6]. As seen in the eye diagrams, the anti-phase VCSEL has a less damped response, which can enhance jitter due to a slow decay of the relaxation oscillations when settling to a new power level. The anti-phase VCSEL also has the largest RMS spectral width $(>0.9 \mathrm{~nm})$, causing much signal degradation due to chromatic and modal fiber dispersion. Dispersion and jitter prevent error-free operation of the antiphase VCSEL at $25 \mathrm{~Gb} / \mathrm{s}$ even over $100 \mathrm{~m} \mathrm{OM3}+$ fiber. The in-phase VCSEL has a more damped response because of the longer photon lifetime. It also has a smaller RMS spectral width $(0.6 \mathrm{~nm})$ and can therefore transmit error-free over $100 \mathrm{~m}$ of fiber. A significant improvement is seen for the SR-VCSEL $(<0.3 \mathrm{~nm}$ RMS spectral width) where error-free transmission at BTB, $100 \mathrm{~m}$ and $300 \mathrm{~m}$ is possible with only a minor power penalty. The SR even enables transmission of $25 \mathrm{~Gb} / \mathrm{s}$ over $500 \mathrm{~m}$ of OM3 + with a $3 \mathrm{~dB}$ power penalty. This is to our knowledge the first time such a large aperture device has been shown to transmit error-free at this high bit rate over as long distance as $500 \mathrm{~m}$. 


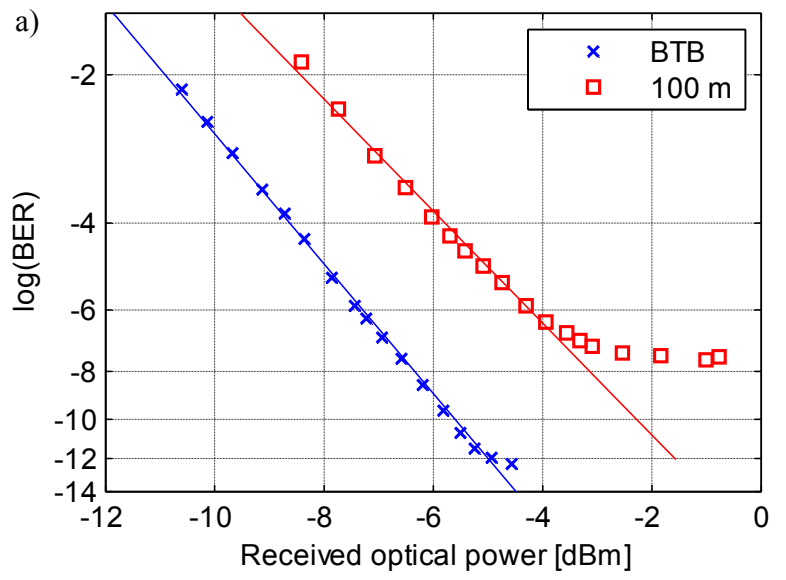

BTB

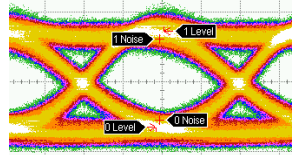

$100 \mathrm{~m}$

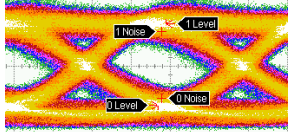

$300 \mathrm{~m}$
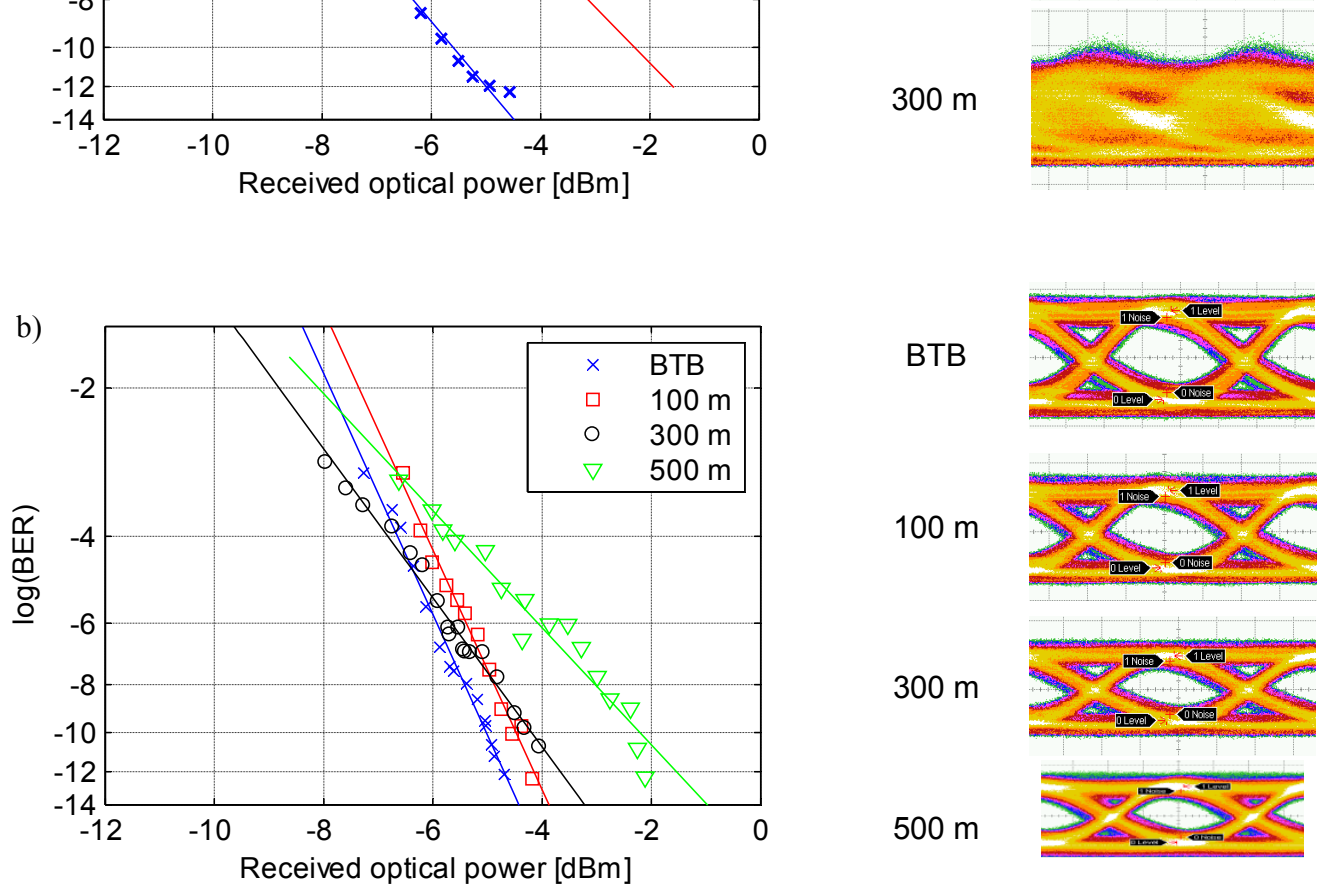

BTB

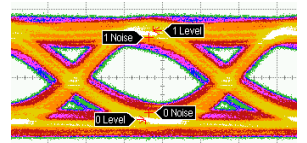

$100 \mathrm{~m}$

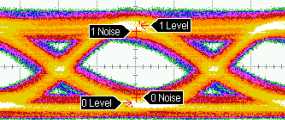

$300 \mathrm{~m}$

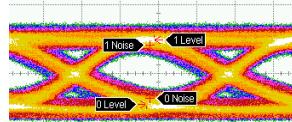

$500 \mathrm{~m}$
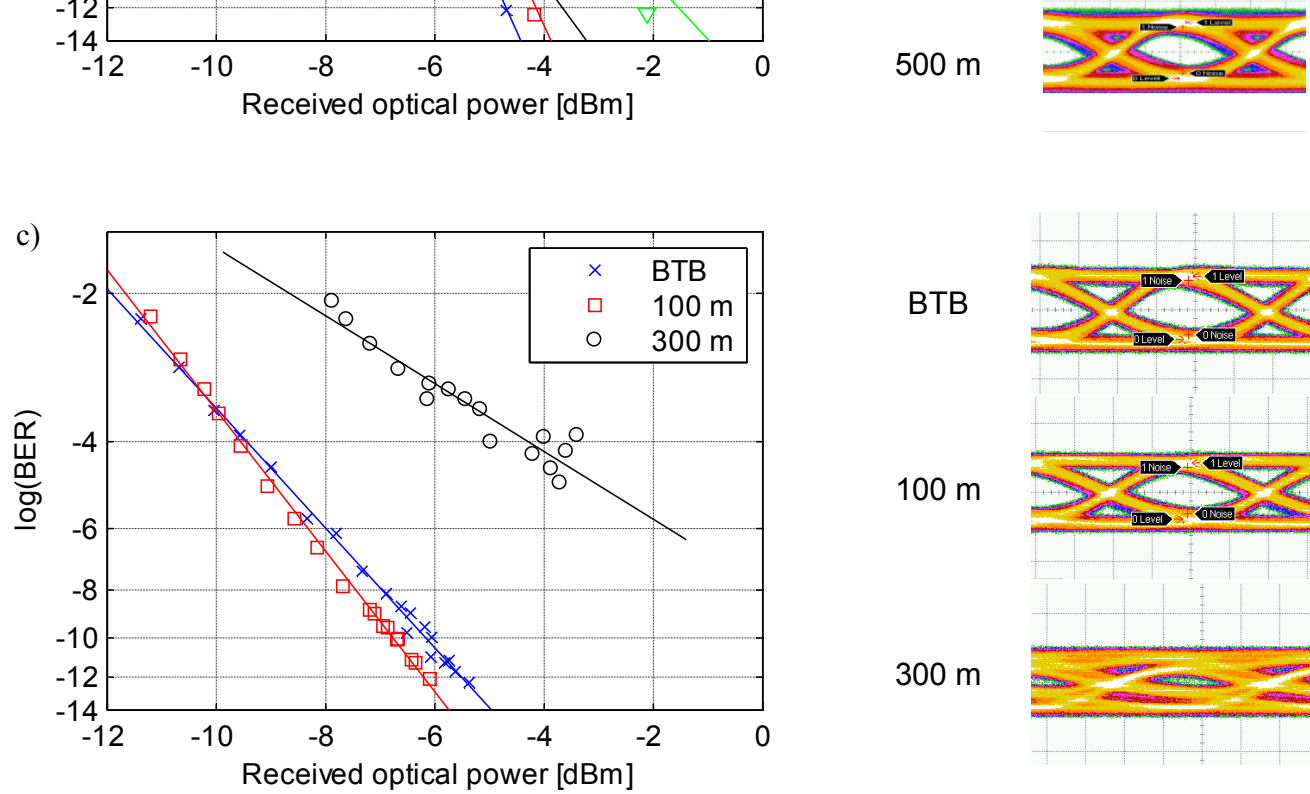

BTB

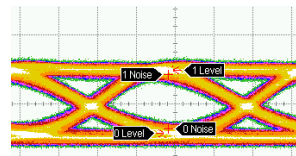

$00 \mathrm{~m}$

$300 \mathrm{~m}$
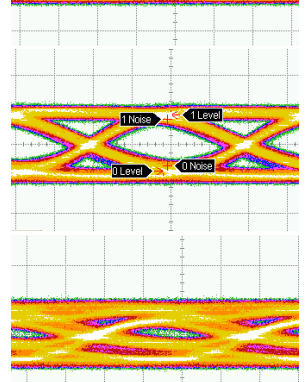

Figure 7. Measured BER versus received power at $25 \mathrm{~Gb} / \mathrm{s}$ with eye diagrams. a) Anti-phase VCSEL, b) SR-VCSEL, c) inphase VCSEL. 


\section{CONCLUSION}

An integrated mode filter, implemented as a shallow surface relief (SR), was investigated with respect to its influence on the high speed properties of $850 \mathrm{~nm}$ oxide confined VCSELs with an oxide aperture of $5 \mu \mathrm{m}$. A significant reduction in RMS spectral width of more than $50 \%$ was observed in comparison with VCSELs without mode filters. The SR-VCSEL complies with current standards (such as IEEE-803.2ae, requiring an RMS spectral width $<0.45 \mathrm{~nm}$ ). Due to the increased damping compared to an anti-phase VCSEL (which has the largest modulation bandwidth), the small signal modulation bandwidth is somewhat reduced, but still amounts to $19 \mathrm{GHz}$. Transmission experiments showed a significant improvement with open eyes and error-free transmission (BER $<10^{-12}$ ) over up to $500 \mathrm{~m}$ of OM3+ fiber, while the anti-phase and in-phase VCSELs enabled error-free transmission only at BTB and up to $100 \mathrm{~m}$ of OM3+ fiber, respectively.

\section{Acknowledgement}

This work has been supported by the European FP7 project VISIT (224211) and the Swedish Foundation for Strategic Research (SSF) project LASTECH.

\section{REFERENCES}

[1] Larsson, A., "Advances in VCSELs for communication and sensing," IEEE J. Sel. Top. Quantum Electron., vol. 17, no. 6, pp. 1552-1567, (2011).

[2] Westbergh, P., et al., "40 Gbis/s error-free operation of oxide-confined $850 \mathrm{~nm}$ VCSEL," Electron. Lett., vol. 46, no. 14, pp. 1014-1016, (2010).

[3] Gholami, A., Molin, D. and Sillard, P., "Compensation of chromatic dispersion by modal dispersion in MMF- and VCSEL-based gigabit ethernet transmissions," IEEE Photon. Techn. Lett., vol. 21, no. 10, pp. 645-647, (2009).

[4] Healy, S. B., et al., "Acitve region design for high-speed 850-nm VCSELs," IEEE J. Quantum Electron., vol. 46, no. 4, pp. 506-512, (2010).

[5] Ou, Y., et al., "Impedance characteristics and parasitic speed limitations of high-speed 850-nm VCSELs," IEEE Photon. Technol. Lett., vol. 21, no. 24, pp. 1840-1842, (2009).

[6] Westbergh, P., et al., "Speed enhancement of VCSELs by photon lifetime reduction," Electron. Lett., vol. 46, no. 13, pp. 938-940, (2010).

[7] Haglund, Å., Gustavsson, J. S., Vukusic, J. A., Modh, P. and Larsson, A., "Single fundamental-mode output power exceeding $6 \mathrm{~mW}$ from VCSELs with a shallow surface relief," IEEE Photon. Technol. Lett., vol. 16, no. 2, pp. 368$370,(2004)$.

[8] Hadley, G. R., "Effective index model for vercial-cavity surface-emitting lasers," Opt. Lett., vol. 20, no. 13, pp. 1483-1485, (1995).

[9] Jung, C., et al., "4.8 $\mathrm{mW}$ singlemode oxide confined top-surface emitting vertical-cavity surface-emitting laser diodes," Electron. Lett., vol. 33, no. 21, pp. 1790-1791, (1997).

[10] Otoma, H., et al., "Single-mode oxide-confined VCSEL for printers and sensors," in IEEE Electronics Systemintegration Technology Conference, Dresden, Germany, pp. 80-85, (2006).

[11] Unold, H. J., Riedl, M. C., Mahmoud, S. W. Z., Jäger, R. and Ebeling, K. J., "Long monolithic cavity VCSELs for high singlemode output power," Electron. Lett., vol. 37, no. 3, pp. 178-179, (2001).

[12] Kardosh, I., Demaria, F., Rinaldi, F., Menzel, S. and Michalzik, R., "High-power single transverse mode verticalcavity surface-emitting lasers with monolithically integrated curved dielectric mirrors," IEEE Photon. Technol. Lett., vol. 20, no. 24, pp. 2084-2086, (2008).

[13] Alias, M. S., Shaari, S., Leisher, P. O. and Choquette, K. D., "Highly confined and continuous single-mode operation of self-aligned photonics crystal oxide VCSEL," Appl. Phys. B., vol. 100, no. 3, pp. 453-459, (2010).

[14] Baveja, P. P., et al., "Impact of device parameters on thermal performance of high-speed oxide-confined 850-nm VCSELs," IEEE J. Quantum Electron., vol. 48, no. 1, pp. 17-26, (2012). 